\title{
Isolated Autoimmune Orchitis Due to IgG4 Hypersecretion Presenting as Tumor-Like Mass: Case Report and Review of Literature
}

\author{
Francisco Marcos da Silva Barroso ${ }^{1}$, Flavio Antunes de Sousa ${ }^{1}$, Paloma Menezes de Souza ${ }^{2,3}$, \\ Gustavo Lopes de Castro ${ }^{*}$, Katia Ramos Moreira Leite ${ }^{5,6}$, Monique Freire Santana ${ }^{7,8,9}$, \\ Roger Arthur da Cunha Alves, Gabriela Ayumi Owada Borges ${ }^{10}$, Juan Eduardo Rios Rodriguez ${ }^{11}$
}

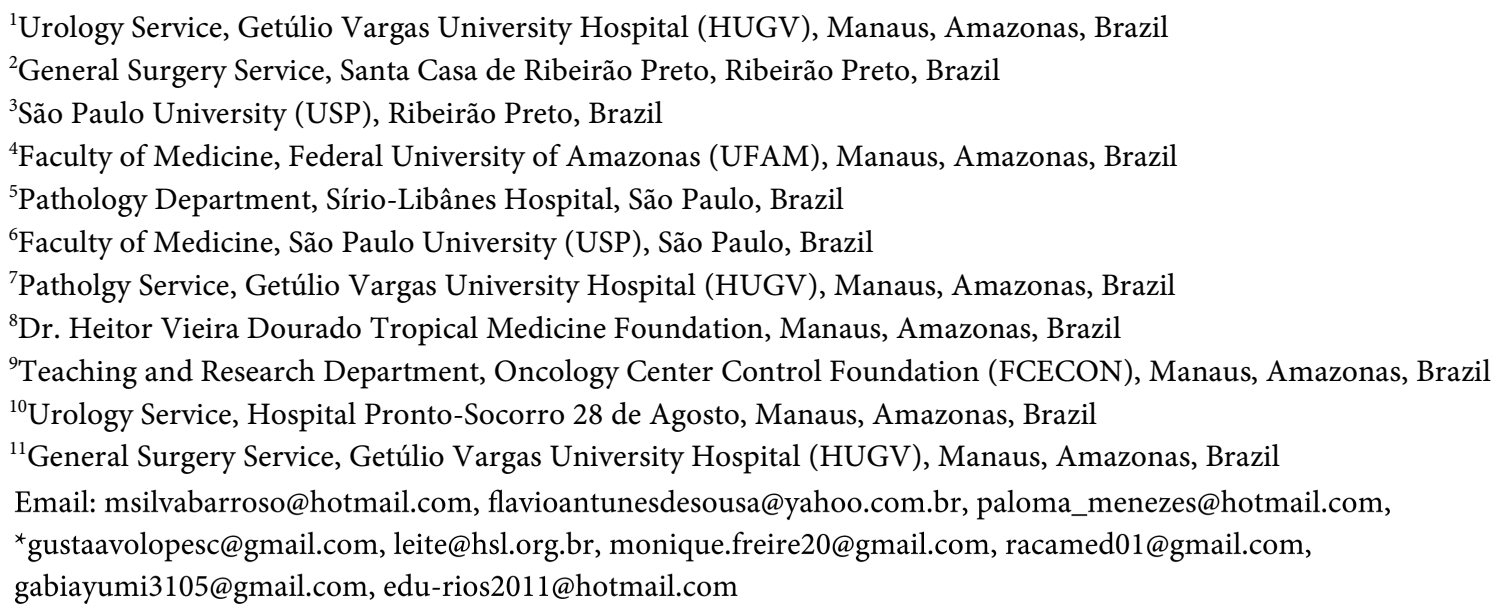

How to cite this paper: da Silva Barroso, F.M., de Sousa, F.A., de Souza, P.M., de Castro, G.L., Leite, K.R.M., Santana, M.F., da Cunha Alves, R.A., Borges, G.A.O. and Rodriguez, J.E.R. (2022) Isolated Autoimmune Orchitis Due to IgG4 Hypersecretion Presenting as Tumor-Like Mass: Case Report and Review of Literature. Open Journal of Urology, 12, 51-56.

https://doi.org/10.4236/oju.2022.121005

Received: October 27, 2021

Accepted: January 14, 2022

Published: January 17, 2022

\begin{abstract}
Background: IgG4-related disease is a rare autoimmune condition that presents with lymphoplasmacytic infiltrate and fibrosis in the organ affected. Isolated testicle involvement is uncommon and there are only a few cases reported in the literature. Case Presentation: We report a case of isolated chronic orchitis due to IgG4 hypersecretion in a 61-year-old patient that evolved with asymptomatic tumor-like mass growth and was treated with left orchiectomy. Histopathological study revealed orchitis related to IgG4 hypersecretion disease. Conclusion: IgG4-related disease can be manifested as a multi or singleorgan disorder. Most diagnoses are made after surgery with histopathological analysis. Most of the cases in literature stand out the difficulty in diagnosis and necessity of high suspicion due to this condition's similarity with neoplasm presentation.
\end{abstract}

\section{Keywords}

Orchitis, IgG4-Related Disease, Autoimmune, Testicle, Inflammatory 
Copyright (C) 2022 by author(s) and Scientific Research Publishing Inc. This work is licensed under the Creative Commons Attribution International License (CC BY 4.0).

http://creativecommons.org/licenses/by/4.0/

\section{Background}

IgG4-related disease is an autoimmune condition that can have a multi-organ or single-organ affection. It is an immune-mediated process due to IgG4 hypersecretion and substantial similarity with other malignant, inflammatory and infectious disorders [1] [2]. It was first associated with autoimmune pancreatitis, however, extra-pancreatic manifestations have been reported [3]. Although in the genitourinary system kidneys, bladder, urethra and prostate gland can be affected, the testicle involvement is rare and was only recently described in 2013 with autoimmune pancreatitis association [4] [5] [6].

IgG4 related orchitis, such as other disorders associated with this condition, is characterized by tumor-like mass growth through lymphoplasmacytic infiltration and fibrosis [1]. It is an uncommon disease with few isolated orchitis cases reported and that requires high suspicion with histopathological correlation.

We report a case of a 61-year-old male patient with chronic orchitis due to IgG4 hypersecretion mimicking testicle tumor, who underwent left orchiectomy.

\section{Key Note Message}

1) Autoimmune orchitis is a rare disease and may present as a tumor-like mass.

2) High suspicion and adequate investigation are necessary for proper diagnosis and treatment.

3) Patients with isolated autoimmune orchitis need orientation for follow-up due to their predisposition for autoimmune conditions.

\section{Case Presentation}

A 61-year-old male patient was admitted to an Amazonas capital's private urology service with a chief complaint of progressive left testicular mass growth for 1 week. He reported painless volume increase with no fever or other infection symptoms association. No drug allergy or cancer history was reported. He also reported healthy previous history without relation to any previous autoimmune condition.

On physical examination, right testicle was topic, unaltered. Left testicle presented with volume increase, hardened aspect and absence of inflammatory signs. A testicle ultrasound with Doppler evidenced volume and vascular flow increase in the left side suggesting inflammatory-infectious process or lymphoproliferative disease. He was treated with non-steroidal anti-inflammatory drugs (NSAIDs) and requested to repeat the ultrasound evaluation. After two weeks, the new testicle ultrasound revealed the same findings, with heterogeneous aspects.

Unfortunately, testis biopsy with frozen section was not available to be performed before orchiectomy. The histopathological analysis revealed orchitis related to IgG4 hypersecretion disease with inflammation process spread throughout the testicle and dense inflammatory infiltrate (Figure 1(A)), predominantly represented by plasmocytes and involving atrophic seminiferous ducts 


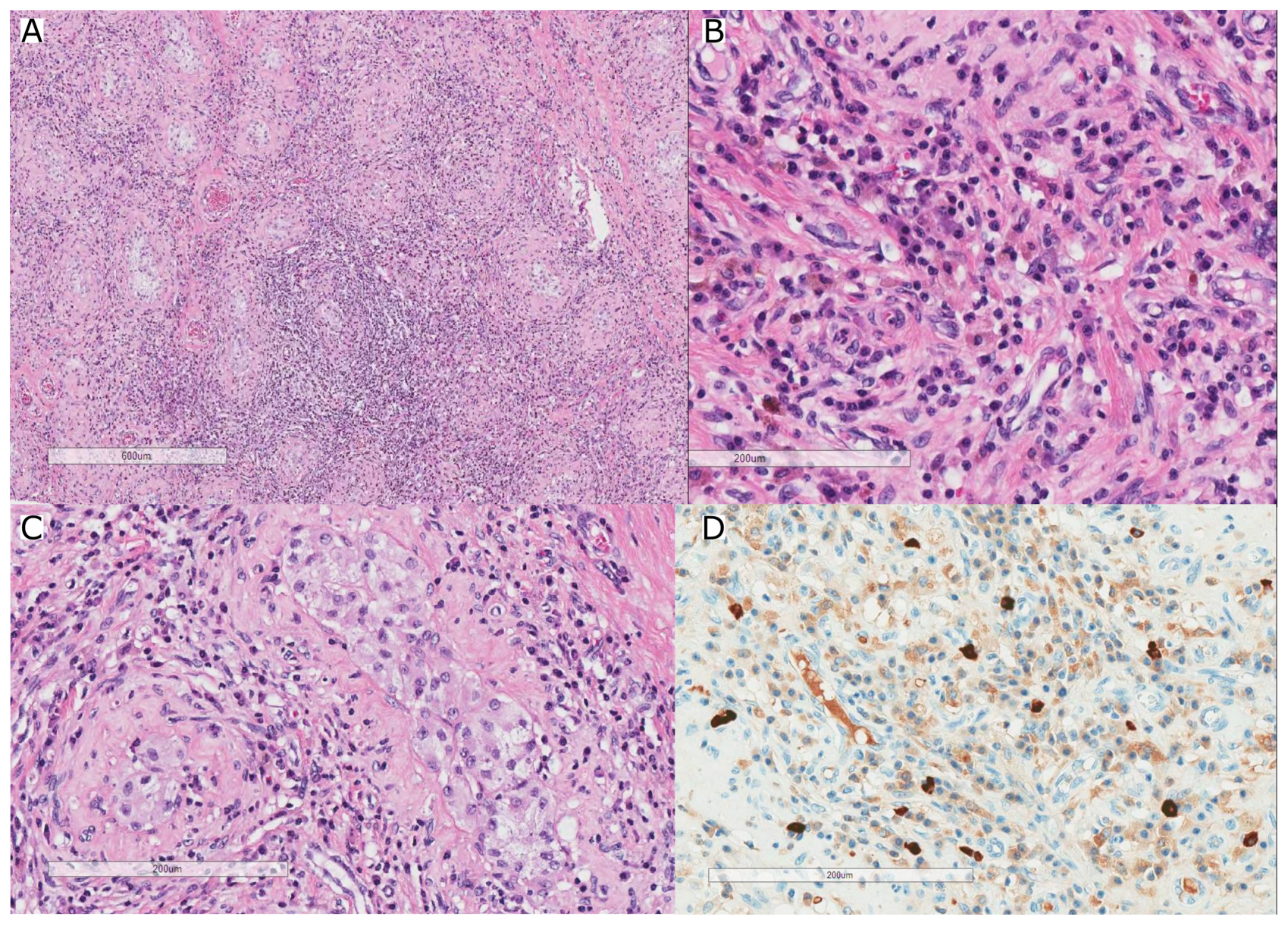

Figure 1. Testicular biopsy consisting of dense inflammatory infiltrate (A); predominantly represented by plasmocytes, ranging from lymphocytes and histiocytes (B); involving atrophic seminiferous ducts, consisting mainly of spermatogonia and exhibiting marked thickening of the basement membrane (C); The immunohistochemistry reaction showed a predominance of IgG4 plasma cells (D).

with spermatogonia and thickening of the basement membrane (Figure 1(C)).

Immunohistochemistry was performed and revealed predominance of IgG4 plasma cells and positivity to B lymphocytes, CD 3, CD 20 and CD 138 (Figures $1(\mathrm{~A})-(\mathrm{D}))$.

In post-operative recovery, the patient evolved without any complications. $\mathrm{He}$ was discharged two days after surgery. In our follow-up, the patient did not present any autoimmune disorder to this moment and was oriented to seek follow-up with rheumatologist and urologist in case of contralateral testicle affection.

\section{Discussion}

IgG4-related disease is a rare condition with systemic or organ-specific affection. It is an immune-mediated disorder with cell marks positive for IgG4 and high serum concentration of this immunoglobulin [1]. IgG4-related disorders affecting testis have only recently been reported in the literature and there is still insufficient epidemiological data, however, most patients are males over 50 years 
old [7] [8] [9].

Although considered an uncommon condition, it has been described as affecting a range of organ systems including the pancreas, liver, aorta, orbits, kidneys, breast and prostate gland [3] [10]. The testicle involvement has recently been reported in 2013 and there are only a few cases of isolated IgG4-related orchitis in the literature [4] [5] [6].

The gold standard for diagnosing chronic orchitis due to IgG4 hypersecretion is a biopsy of the affected tissue with immunohistochemical analysis. IgG4 serum evaluation up to $135 \mathrm{mg} / \mathrm{dL}$ also suggests the diagnosis, but normal serum levels do not exclude it once $30 \%-40 \%$ of the cases revealed normal values at the moment of diagnosis. However, serum IgG4 monitoring is useful for follow-up treatment, and its serum concentration tends to rise with systemic affection [5]. A 2012 consensus statement on pathological criteria for the diagnosis of IgG4-related disease has been established and includes the presence of an increased number of IgG4 plasma cells associated with lymphoplasmacytic infiltration, fibrosis, and obliterated phlebitis [11]. Immunohistochemistry may be positive for B cells and their subtypes, IgG4, CD 68 and CD 20, which prolongs the disease through continuous antigens presentation [12].

IgG4-related disorders' pathophysiology has not been clearly described, but IgG4 is responsible for modulating the response against infectious agents and allergic components. In these conditions, the T CD4 cell sensitization activates innate immune cells such as fibroblasts and naïve B cell entrance in the tissue, responsible for the fibrosis and continuous inflammatory process [8].

The treatment for these IgG4 hypersecretion conditions is not firmly stablished, although most clinical manifestations respond to corticoids. For this reason, these drugs are usually the treatment of choice for most patients [1] [4]. Glucocorticoids seem to have low immunoglobulin levels and the disease start remission. Recently, rituximab was introduced as an option treatment for these conditions [8] [13].

Nevertheless, orchiectomy is opted in most cases due to difficulty differentiation with malignant neoplasm and IgG4-related being diagnosed only after histopathological analysis [10].

As evidenced in our case, the affection of testis by IgG4 hypersecretion causes organ volume increase with variable consistency (fibro-elastic or hardened), usually unilateral and painful, which differs from our report since the patient evolved painlessly. Many patients are treated with antibiotics and analgesics for infectious hypotheses, without any clinical improvement [14].

In our case, the patient presented isolated orchitis as the main manifestation of IgG4-related disease. Although it is important to keep in mind that other organs affection may happen in a synchronous or metachronous way [8]. This patient follow-up was brief and he evolved asymptomatic.

\section{Conclusion}

The IgG4-related disease is an uncommon condition associated with autoim- 
mune relations and cell sensitization. Isolated testicle affection is infrequent and it clinically presents as chronic orchitis and mimicking tumor-like mass. Most cases are diagnosed after surgery with histopathological analysis, indicating that a high suspicious index and proper investigation are necessary.

\section{Ethics Approval and Consent to Participate}

This study was exempt from ethical approval.

\section{Consent for Publication}

Informed consent was obtained in both written and verbal forms from the patient to publish this case report and the images on it.

\section{Conflicts of Interest}

The authors declare that they have no competing interests.

\section{References}

[1] Kamisawa, T., Zen, Y., Pillai, S. and Stone, J. (2015) IgG4-Related Disease. Lancet, 385, 1460-1471. https://doi.org/10.1016/S0140-6736(14)60720-0

[2] Mahajan, V., Mattoo, H., Deshpande, V., Pillai, S. and Stone, J. (2014) IgG4-Related Disease. Annual Review of Pathology, 9, 315-347.

https://doi.org/10.1146/annurev-pathol-012513-104708

[3] Stone, J., Zen, Y. and Deshpande, V. (2012) IgG4-Related Disease. The New England Journal of Medicine, 366, 539-551. https://doi.org/10.1056/NEJMra1104650

[4] Lal, J., Bhat, S., Doddamani, S. and Devi, L. (2016) Isolated Testicular Immunoglobulin G4-Related Disease: A Mimicker of Malignancy. Indian Journal of Urology, 32, 326-328. https://doi.org/10.4103/0970-1591.191273

[5] Shams, A., DAS, A., Sinha, M., et al. (2021) IgG4-Related Disease with Selective Testicular Involvement-A Rare Entity: Case Report with Review of Literature. Turkish Journal of Pathology, 37, 78-83. https://doi.org/10.5146/tjpath.2020.01493

[6] de Buy Wenniger, L., Scheltema, J., Verheij, J. and Beuers, U. (2013) Testicular Inflammation as a New Manifestation of IgG4-Associated Disease. Urology, 82, e15-e16. https://doi.org/10.1016/j.urology.2013.04.046

[7] Zen, Y. and Nakanuma, Y. (2010) IgG4-Related Disease: A Cross-Sectional Study of 114 Cases. The American Journal of Surgical Pathology, 34, 1812-1819. https://doi.org/10.1097/PAS.0b013e3181f7266b

[8] Opriță, R., Opriță, B., Berceanu, D. and Diaconescu, I. (2017) Overview of IgG4Related Disease. Journal of Medicine and Life, 10, 203-207.

[9] Lang, D., Zwerina, J. and Pieringer, H. (2016) IgG4-Related Disease: Current Challenges and Future Prospects. Therapeutics and Clinical Risk Management, 12, 189199. https://doi.org/10.2147/TCRM.S99985

[10] Karram, S., Kao, C., Osunkoya, A., Ulbright, T. and Epstein, J. (2014) Idiopathic Granulomatous Orchitis: Morphology and Evaluation of Its Relationship to IgG4 Related Disease. Human Pathology, 45, 844-850. https://doi.org/10.1016/j.humpath.2013.12.003

[11] Deshpande, V., Zen, Y., Chan, J., et al. (2012) Consensus Statement on the Pathology of IgG4-Related Disease. Modern Pathology, 25, 1181-1192. 
[12] Migita, K., Miyashita, T., Mizuno, A., et al. (2014) IgG4-Related Epididymo-Orchitis Associated with Bladder Cancer: Possible Involvement of BAFF/BAFF-R Interaction in IgG4-Related Urogenital Disease. Modern Rheumatology, 24, 188-194. https://doi.org/10.3109/14397595.2013.852841

[13] Khosroshahi, A., Wallace, Z., Crowe, J., et al. (2015) Second International Symposium on IgG4-Related Disease. International Consensus Guidance Statement on the Management and Treatment of IgG4-Related Disease. Arthritis \& Rheumatology, 67, 1688-1699. https://doi.org/10.1002/art.39132

[14] Roy, S., Hooda, S. and Parwani, A. (2011) Idiopathic Granulomatous Orchitis. Pathology-Research and Practice, 207, 275-278.

https://doi.org/10.1016/j.prp.2011.02.005

\section{List of Abbreviations}

NSAIDs: Non-steroidal anti-inflammatory drugs;

IgG4: Immunoglobulin G type 4;

CT: Computed tomography. 